\title{
COVID-19 and the brain: impact on nuclear medicine in neurology
}

\section{Silvia Morbelli ${ }^{1,2} \cdot$ Ozgul Ekmekcioglu $^{3} \cdot$ Henryk Barthel $^{4} \cdot$ Nathalie L. Albert $^{5} \cdot$ Ronald Boellaard $^{6} \cdot$ Diego Cecchin $^{7}$. Eric Guedj ${ }^{8}$. Adriaan A. Lammertsma ${ }^{6} \cdot$ Ian Law $^{9} \cdot$ Ivan Penuelas $^{10} \cdot$ Franck Semah $^{11} \cdot$ Tatjana Traub-Weidinger $^{12}$. Elsmarieke van de Giessen ${ }^{13}$. Andrea Varrone ${ }^{14}$. Valentina Garibotto ${ }^{15,16}$. EANM Neuroimaging Committee}

Published online: 23 July 2020

(C) Springer-Verlag GmbH Germany, part of Springer Nature 2020

\section{Introduction}

On 11 March 2020, the World Health Organization (WHO) declared the outbreak of coronavirus disease 2019 (COVID19) to be a pandemic [1]. The wide spread of infections led to an immediate urgency with the number of affected patients exceeding capacities of many involved healthcare systems with substantial morbidity and mortality [2].

During the pandemic emergency, hospitals and healthcare facilities all over the world were forced to postpone elective procedures. Thankfully, in keeping with different stages and timeframe of the curve of infection, it has been possible to progressively restart these activities over time in several countries [3]. Both during the pandemic crisis and in the present

This article is part of the Topical Collection on Infection and Inflammation

Silvia Morbelli

silviadaniela.morbelli@hsanmartino.it

1 IRCCS Ospedale Policlinico San Martino, Genoa, Italy

2 Nuclear Medicine Unit, IRCCS Ospedale Policlinico San Martino, Department of Health Sciences(DISSAL), University of Genoa, Genoa, Italy

3 Department of Nuclear Medicine, Sisli Etfal Education and Research Hospital, Istanbul, Turkey

4 Department of Nuclear Medicine, Leipzig University, Leipzig, Germany

5 Department of Nuclear Medicine, Ludwig Maximilians-University of Munich, Munich, Germany

6 Department of Radiology and Nuclear Medicine, Amsterdam UMC, Location VUmc, De Boelelaan, 1117 Amsterdam, Netherlands

7 Nuclear Medicine Unit, Department of Medicine - DIMED, University of Padua, Padua, Italy

8 APHM, CNRS, Centrale Marseille, Institut Fresnel, Timone Hospital, CERIMED, Nuclear Medicine Department, Aix Marseille Univ, Marseille, France transition phase, measures and precautions have been particularly relevant for fragile patients [3, 4]. In fact, comorbidities together with age have been demonstrated as important prognostic factors for the severity of disease in patients affected by SARS-CoV-2 [2, 5].

Among fragile subjects, patients with chronic neurological disease are more susceptible to the effects of the infection, particularly involving the respiratory tract $[6,7]$. Impairment of central respiratory centres (CNS) could also contribute to the severe acute respiratory syndrome associated with severe acute respiratory syndrome coronavirus 2 (SARS-CoV-2) [8].

Given the high impact of these disorders on human health, there is a present need to progressively restore necessary access and care for patients affected by neurological and

9 Department of Clinical Physiology, Nuclear Medicine and PET, Rigshospitalet, University of Copenhagen, Copenhagen, Denmark

10 Department of Nuclear Medicine, Clinica Universidad de Navarra, IdiSNA, University of Navarra, Pamplona, Spain

11 Nuclear Medicine Department, University Hospital, Lille, France

12 Division of Nuclear Medicine, Department of Biomedical Imaging and Image-guided Therapy, Medical University of Vienna, Vienna, Austria

13 Radiology and Nuclear Medicine, Amsterdam UMC, Location AMC, Meibergdreef 9, Amsterdam, The Netherlands

14 Centre for Psychiatry Research, Department of Clinical Neuroscience, Karolinska Institutet \& Stockholm Health Care Services, Region Stockholm, Stockholm, Sweden

15 NIMTLab, Faculty of Medicine, Geneva University, Geneva, Switzerland

16 Division of Nuclear Medicine and Molecular Imaging, Geneva University Hospitals, Geneva, Switzerland 
psychiatric diseases, including molecular imaging techniques which are part of the diagnostic work-up of these patients [4].

The organization of nuclear medicine (NM) services during the pandemic and the present progressive transition to normality (or to a "new normal") have been analysed and previously discussed by international groups of experts endorsed by the International Atomic Energy Agency (IAEA), European Association of Nuclear Medicine (EANM) and Society of Nuclear Medicine and Molecular Imaging (SNMMI) [3, 4, 9].

In the present transition phase, restored availability of brain PET and SPECT examinations should be organized following local, national and international indications for NM procedures. However, the peculiar characteristics of patients with neurological diseases, the present knowledge of neuroCOVID features and potentially sequelae, together with the concomitant effect of the pandemic emergency and the lockdown on patients with neurodegenerative and psychiatric diseases should be taken into account when resuming nuclear neurology activity $[6,10]$.

Finally, during the COVID-19 emergency, in several countries, clinical trials (including interventional trials on new disease-modifying drugs for neurological diseases such as Alzheimer's disease, AD) have temporarily been suspended, possibly further delaying the identification of new treatments (crucial for patients and inevitably impacting the final validation of all imaging biomarkers in the clinical settings) $[11,12]$. There, the EANM Neuroimaging Committee will discuss potential challenges related to neurological manifestations and complications of COVID-19 as well as the upcoming implications of the COVID-19 outbreak for patients with neurological diseases, who were candidates for brain PET and SPECT both in clinical and research settings.

\section{Neurological manifestations and complications of COVID-19: implications for nuclear medicine}

Coronavirus infections have been associated with neurological manifestations, like febrile seizures, convulsions, change in mental status and encephalitis, and neuro-invasive capabilities of coronaviruses have been described in humans [2]. Indeed, SARS-CoV-2 acts on angiotensin-converting enzyme- 2 receptors that are expressed in lung alveoli type 2 as well as in the brain (mainly in brainstem) [13, 14]. In this framework, neuro-invasion of SARS-CoV2 has even been advocated to explain the development of respiratory failure in some patients [13-15].

While some patients with COVID-19 may show confusion and headache (non-specific neurological symptoms), others may develop specific neurological manifestations including stroke, seizures and signs of encephalitis [16].
From the pathophysiological point of view, upon nasal infection, coronavirus has been demonstrated to enter the CNS through the olfactory bulb $[17,18]$. In this framework, especially during the spread of the infection in European countries, a peculiar presentation of COVID-19 has been described as characterized by olfactory and gustatory dysfunctions [16]. These symptoms may appear before, during or even after the general symptoms $[17,18]$.

Smell dysfunction obviously occurs rather often in viral infections [19], as many viruses may cause inflammatory reaction of the nasal mucosa and subsequent rhinorrhoea [19]. However, olfactory dysfunction linked to COVID-19 infection is peculiar as it is not associated with rhinorrhoea suggesting that nasal inflammation may not be the only underlying etiological factor $[17,20]$. In this context, the pathophysiological mechanisms leading to olfactory and gustatory dysfunctions in the COVID-19 infection are still unknown. Understanding the potential relationship between invasion of the olfactory bulb by SARS-CoV-2 and involvement of the CNS is an area of interest for future research to improve knowledge of the pathophysiology of the disease.

Another manifestation linked to the pathophysiology of COVID-19 is the association with coagulopathy and increase of thrombotic events. Initially, there were reports of increased stroke incidence in COVID-19 although these might have been overestimated [21]. However, stroke in COVID-19 patients is generally more severe and may occur in younger patients [21]. Nuclear imaging usually is not applied in stroke, but post-stoke abnormalities may be present as unexpected incidental findings complicating the interpretation of nuclear imaging studies performed for other indications.

Finally, although neuro-COVID-19 has been increasingly described, not only mechanisms of action but also frequency and severity of CNS symptoms, and their relationship with the course and severity of COVID-19 still needs to be elucidated.

Brain scanning is not a priority in imaging algorithm of COVID-19 and might even be not possible for several patients. Magnetic resonance imaging (MRI) may play a role in a subgroup of patients during the acute phase and might help to understand mechanisms of neuro-COVID also in the immediate post-recovery phase [22-24]. However, normal morphology of the olfactory bulb on MRI has been reported in COVID-19 patients with anosmia [25, 26]. In other small case series, cortical hyperintensity on fluid-attenuated inversion recovery images in the gyrus rectus and in the olfactory bulbs has been reported [26]. However, these alterations were evident in the very early course of the disease but completely disappeared at early follow-up (less than 1 month after symptoms onset) [26].

Accordingly, the question can be raised whether decreased neural activity could be demonstrated in olfactory pathways in 
patients with normal morphology and, if so, which is the time course of functional alterations.

Karimi-Galougahi et al. reported a case of a patient affected by COVID-19 with a persistent isolated anosmia for 6 weeks with a normal brain MRI [27]. The patient was submitted to resting $2-\left[{ }^{18} \mathrm{~F}\right] \mathrm{FDG}$ PET, and the resulting images highlighted hypometabolism of the left orbitofrontal cortex, which indeed is involved (together with the limbic system) in the chemosensory process [27]. Using PET, Guedj et al. confirmed metabolic impairment of the olfactory/rectus gyrus in two patients with SARS-CoV-2 by means of quantitative analysis in comparison with healthy controls [28]. In one patient, an extension into the medial temporal cortex and the brainstem was also highlighted [28].

While these findings sound plausible from the pathophysiological point of view, with also the possible secondary impact of brain inflammation on various neurological and psychiatric disorders, no other PET or SPECT data are available in patients with COVID-related olfactory dysfunction. It should be noted, however, that patients with hypo and anosmia due to CNS damage of other aetiologies have been extensively investigated by means of molecular imaging in recent years $[29,30]$.

In particular, hyposmia is one of the most common and best-characterized non-motor features of Parkinson disease (PD), and a large body of evidence demonstrates that prodromal individuals with hyposmia, who later convert to clinical PD, already show altered availability of dopamine transporters [29, 30]. This SPECT finding strongly increases specificity of early diagnosis and retains the high sensitivity associated with olfactory testing alone [28, 29]. Notably, very preliminary data related to the co-expression and regulation of ACE2 and DOPA Decarboxylase (DDC) genes have raised the hypothesis that an alteration of the dopamine synthetic pathways is possibly involved in the pathophysiology of COVID-19 [31]. Following this hypothesis, as SARS-CoV-2 induces downregulation of ACE2 expression, it might also result in alterations of the dopamine (and serotonin) synthetic pathways that might be the target for future investigation, especially just after patients' recovery, in selected centres with appropriate resources for clinical research and follow-up of survivors of COVID-19 [31].

Finally, while mid-term and long-term consequences of SARS-CoV-2 neuro-infection are still unknown, cases of post-COVID-19 likely autoimmune (steroid responsive) but seronegative encephalitis have been reported in the absence of any alteration on MRI [32]. While causality and pathophysiology of these manifestations still need to be elucidated, NM physicians should be aware of this possibility as it might result in an indication for $2-\left[{ }^{18} \mathrm{~F}\right] \mathrm{FDG}$ PET as suggested in the emerging diagnostic work-up of autoimmune encephalitis $[33,34]$.

\section{Impact of COVID-19 outbreak on patients with neurological disorders}

As mentioned, another area of concern is the vulnerability of patients with neurological diseases to both COVID-19 and to the related social distance measures. First of all, it might be difficult for people living with dementia to remember selfquarantine and protection measures (such as wearing masks), thus being exposed to higher risks of infection [10]. Moreover, behavioural symptoms of dementia, such as wandering, may hamper efforts to maintain isolation [10]. Individuals with dementia are more likely to have cardiovascular disease, metabolic syndrome and pneumonia with respect to non-demented elderly subjects, thus adding the cumulative effect of other comorbidities to the risk of a poor outcome after COVID-19 infection [7]. Moreover, stress and trauma can accelerate cognitive decline in patients with dementia, while depression due to both COVID-19 pandemic and social distancing measures may favour onset of cognitive symptoms or even pseudodementia that might represent a source of challenging differential diagnosis in the present context [35]. Indeed, the differentiation between mild cognitive impairment (MCI), or dementia, due to depression and MCI due to $\mathrm{AD}$ represents a typical indication for brain 2-[ $\left[{ }^{18}\right.$ F]FDG PET [36-39].

Similarly, PD can compromise the respiratory system, and patients with advanced PD are known to have increased risk of pneumonia [40]. Accordingly, at least in theory, PD might be a risk factor for more severe respiratory complications or even an unfavourable outcome after COVID-19 infection [41]. However, the relationship between PD pathophysiology and SARS-CoV-2 might be even more intricate as a possible protective role of alpha-synuclein against the spread of the disease from peripheral to CNS has been reported [42]. This unexpected finding might lead to a search for a better understanding of the physiological role of alpha-synuclein in immune response and inflammatory conditions, which might be relevant for research on PD pathogenesis in the future.

On the other side, it has been suggested that increased stress due to the pandemic outbreak may further unmask a latent hypokinetic rigid syndrome, probably by depleting compensatory mechanisms in PD patients $[6,43]$. This might even lead to an increase in numbers of new PD diagnoses just after the pandemic crisis.

These challenges might potentially increase the need for medical care for patients with neurological diseases in the coming months. Moreover, as it is well-known that during the pandemic outbreak, the increased demand on health systems has resulted in the diversion of resources away from patients with all types of chronic diseases, the progressive resolution of the pandemic is likely to be associated with an increased demand for diagnosis and care that so far has been deferred [44]. 
The workflow of NM departments during the first phase of the pandemic outbreak has been modified to focus on more urgent examinations. This has, in many cases, resulted in a significant reduction in the number of brain PET and SPECT examinations in patients with chronic neurological diseases. This organizational change has also resulted in reduced access of fragile patients with neurological disorders, in an attempt to protect them by avoiding access to hospitals during the pandemic emergency. However, the same group of patients might now suffer from a slower restart of the availability of NM procedures for diagnostic work-up of $\mathrm{AD}, \mathrm{PD}$ or epilepsy for several logistic reasons, not at last for potential scarcity of resources to be allocated.

As a matter of fact, the consequences of the pandemic go well beyond the peak period. When resuming to normality and establishing priorities for the many societal and economical challenges ahead, it will be important to avoid the equation "non-urgent $=$ non-essential $=$ futile". Accordingly, the risk $/$ benefit ratio for performing brain PET and SPECT studies in such type of fragile patients should progressively change following local scenario and authority's indications but should also be tailored more than ever to individual bases and patients' diagnostic needs.

The procedures to secure safe patient examinations in the present transition phase in European Countries are now in place and should not prevent examinations. In countries with a developed social sector, any delay in patient assessment will withhold social support and benefits that are contingent on an accurate diagnosis with potentially negative consequences for the patient. Neurodegenerative disorders are among the most difficult and challenging diagnosis, and PET and SPECT brain imaging are important supplementary imaging modalities, which are increasingly being used and relied on.

\section{Clinical trials involving brain PET in the era of COVID-19 pandemic}

One of the collateral damages associated with the COVID-19 pandemic has also been the interruption of clinical trials and observational studies using brain PET as diagnostic and response monitoring marker. This is particularly true for research in the field of $\mathrm{AD}$ and other neurodegenerative conditions, occurring in elderly populations vulnerable for COVIDrelated complications [35] (https://www.alzforum.org/news/ community-news/alzheimers-research-restarts-cautiously).

This interruption is due to a number of reasons. In multiple countries and states, there has been a global halt of non-urgent/less-urgent medical procedures, which by definition included research studies, and a general population lockdown. For observational studies, the evaluations of the risk-benefit balance have overall concluded that the risks associated with the participation in the study are higher than the benefits in understanding the disease progression and evolution that can be obtained with the studies. In case of clinical trials, for which direct benefit can be expected for participants in the study, the limited availability of health resources and organizational and economic issues in pharmaceutical companies have probably been determinant for decisions related to the manage ongoing clinical trials.

Some studies have been able to, at least, partly continue with a robust shift to remote rather than in-person visits whenever possible. This change, enabled by technology, might have positive consequences in the longer-term by facilitating screening and follow-ups and thus access to trials and results monitoring [45]. The COVID-19 pandemic could even accelerate the adoption of innovative design and methods in clinical research.

Moreover, the policy to halt research studies during the peak phase of the disease is well-understandable and in line with the global halt of socio-economical activities. However, the effect of the pandemic outbreak on research may also "threaten the patients of tomorrow" (see reference 35 for a more detailed discussion). We all hope that the fact that many countries are restarting non-urgent activities and trials will prove to be a success and ultimately the COVID-related stop will only result in a few months delay.

In fact, these consequences may be even more relevant for the field of research in neurodegenerative dementia and in particular in $\mathrm{AD}$. In the last 20 years, the field of dementia has become one of the most active areas for both basic and clinical research. The identification of new potentially disease-modifying drugs has been supported by huge efforts by major industry and governments [46]. An interruption of these important efforts could also have long-lasting consequences for the field of $\mathrm{AD}$ research and future clinical management. A recent report of the Alzheimer's forum has discussed the impact on AD clinical trials, confirming that a number of trials were terminated during the pandemic crisis. Moreover, guidelines are missing on how to adjust statistical analyses especially considering that some patients declined more easily during the pandemic, and trial recruitment slowed [47]. Finally, sponsored and no-profit researches have deadlines, and although no-cost extension is possible (especially given the present circumstances), research contracts (including funds to cover $\mathrm{PhD}$ fees) might finish before the trial is completed raising the need for additional expenses.

As a matter of fact, the dementia scientific community might even face more challenges for funds and social awareness in the next future. It is important to keep in mind that clinical research, also outside the research dedicated to the fight of the COVID-19, is an essential pillar of our societies and health systems. The Nuclear Medicine scientific community will have to deploy all its lobbying capacity to minimize 
the negative impact of the global economic crises on funding research on molecular imaging biomarkers.

\section{Conclusions}

Although the underlying pathophysiology of neurological manifestations in SARS-CoV-2 remains to be fully determined, an increasing number of studies report neurological involvement and complications in patients with COVID-19. While progressively resuming nuclear neurology activity in the present transition phase, NM physicians should be aware of these possible clinical presentations as well as of the expected relevant impact of both COVID-19 and social distancing on patients with neurological and psychiatric diseases. During the pandemic crisis, the justification principle has been applied more than ever for the decision-making about all imaging procedures (especially for elderly patients with comorbidities).

In the present transition phase, the risk/benefit ratio for performing brain PET and SPECT scans in fragile patients should be tailored to individual bases with a thoughtful consideration also for patients' diagnostic needs. Finally, the pandemic has clearly demonstrated how much the whole integrity of our community relies on health systems and their proper functioning. In this framework, clinical research is an intrinsic component of our health systems that should also be supported to mitigate long-lasting impact of COVID-19, also for patients with chronic neuropsychiatric diseases still lacking effective treatments.

Authors' contribution All authors were involved in the manuscript writing and revision.

\section{Compliance with ethical standards}

Conflict of interest SM received speaker honoraria from GE Healthcare. Ronald Boellaard has provided data analysis service for Bristol Meyers Squibb, Rodin and Roche and is a scientific advisor for EARL; AAL has no conflicts of interest. Ian Law has received speaker honoraria from Siemens Healthcare. IP received research funding from Vivet Therapeutics. VG received research/teaching funding from Siemens Healthineers, GE Healthcare, Roche, Merck and Life Molecular Imaging. All the other authors declare no conflict of interest.

Guarantor Not applicable.

Statistics and biometry Not applicable.

Informed consent Not applicable.

Ethical approval Institutional Review Board approval was not required because the paper is an editorial.

\section{Study subjects or cohorts overlap Not applicable.}

Methodology Not applicable.

\section{References}

1. Bassetti M, Vena A, Giacobbe DR. The novel Chinese coronavirus (2019-nCoV) infections: challenges for fighting the storm. Eur J Clin Investig. 2020;50:e13209.

2. Zhou F, Yu T, Du R, et al. Clinical course and risk factors for mortality of adult inpatients with COVID-19 in Wuhan, China: a retrospective cohort study. Lancet. 2020;395:1054-62.

3. Paez D, Gnanasegaran G, Fanti S, et al. COVID-19 pandemic: guidance for nuclear medicine departments. Eur J Nucl Med Mol Imaging. 2020;47:1615-9.

4. Huang HL, Gnanasegaran G, Paez D, et al. Nuclear medicine services after COVID-19: gearing up back to normality. Eur J Nucl Med Mol Imaging. 2020:1-6.

5. Abbatecola AM, Antonelli-Incalzi R. Editorial: COVID-19 spiraling of frailty in older Italian patients. J Nutr Health Aging. 2020;24: 453-5.

6. Helmich RC, Bloem BR. The impact of the COVID-19 pandemic on Parkinson's disease: hidden sorrows and emerging opportunities. J Park Dis. 2020;10:351-4.

7. Bauer K, Schwarzkopf L, Graessel E, et al. A claims data-based comparison of comorbidity in individuals with and without dementia. BMC Geriatr. 2014;14.

8. Leonardi M, Padovani A, McArthur JC. Neurological manifestations associated with COVID-19: a review and a call for action. J Neurol. 2020;267:1573-6.

9. SNMMI COVID-19 Resource Center. J Nucl Med. 2020;61:11N

10. Wang H, Li T, Barbarino P, et al. Dementia care during COVID-19. Lancet. 2020;395:1190-1.

11. Fleming TR, Labriola D, Wittes J. Conducting clinical research during the COVID-19 pandemic: protecting scientific integrity. JAMA. 2020. https://doi.org/10.1001/jama.2020.9286.

12. Deroose CM, Lecouvet FE, Collette L, et al. Impact of the COVID19 crisis on imaging in oncological trials. Eur J Nucl Med Mol Imaging. 2020:1-5.

13. Huang YH, Jiang D, Huang JT. SARS-CoV-2 detected in cerebrospinal fluid by PCR in a case of COVID-19 encephalitis. Brain Behav Immun. 2020;87:149.

14. Desforges M, Le Coupanec A, Stodola JK, et al. Human coronaviruses: viral and cellular factors involved in neuroinvasiveness and neuropathogenesis. Virus Res. 2014;194: $145-58$.

15. Li YC, Bai WZ, Hashikawa T. The neuroinvasive potential of SARS-CoV2 may play a role in the respiratory failure of COVID19 patients. J Med Virol. 2020;92:552-5.

16. Asadi-Pooya AA, Simani L. Central nervous system manifestations of COVID-19: a systematic review. J Neurol Sci. 2020;413: 116832.

17. Lechien JR, Chiesa-Estomba CM, De Siati DR, et al. Olfactory and gustatory dysfunctions as a clinical presentation of mild-tomoderate forms of the coronavirus disease (COVID-19): a multicenter European study. Eur Arch Otorhinolaryngol. 2020:1-11.

18. Bénézit F, Le Turnier P, Declerck C, et al. Utility of hyposmia and hypogeusia for the diagnosis of COVID-19. Lancet Infect Dis. 2020:S1473-3099;30297-8.

19. Suzuki M, Saito K, Min WP, Vladau C, Toida K, Itoh H, et al. Identification of viruses in patients with postviral olfactory dysfunction. Laryngoscope. 2007;117:272-7.

20. van Riel D, Verdijk R, Kuiken T. The olfactory nerve: a shortcut for infuenza and other viral diseases into the central nervous system. J Pathol. 2015;235:277-87.

21. Tsivgoulis G, Katsanos AH, Ornello R, Sacco S. Ischemic stroke epidemiology during the COVID-19 pandemic: navigating uncharted waters with changing tides. Stroke. 2020;51:1924-6. 
22. Radmanesh A, Raz E, Zan E, Derman A, Kaminetzky M. Brain imaging use and findings in COVID-19: a single academic center experience in the epicenter of disease in the United States [published online ahead of print, 2020 May 28]. AJNR Am J Neuroradiol. 2020. https://doi.org/10.3174/ajnr.A6610.

23. Kandemirli SG, Dogan L, Sarikaya ZT, et al. Brain MRI Findings in Patients in the Intensive Care Unit with COVID-19 Infection [published online ahead of print, 2020 May 8]. Radiology. 2020: 201697.

24. Poyiadji N, Shahin G, Noujaim D, Stone M, Patel S, Griffith B. COVID-19-associated Acute Hemorrhagic Necrotizing Encephalopathy: CT and MRI Features [published online ahead of print, 2020 Mar 31]. Radiology. 2020:201187.

25. Galougahi MK, Ghorbani J, Bakhshayeshkaram M, Naeini AS, Haseli S. Olfactory bulb magnetic resonance imaging in SARSCoV-2-induced anosmia: the first report. Acad Radiol. 2020;27: $892-3$.

26. Politi LS, Salsano E, Grimaldi M. Magnetic resonance imaging alteration of the brain in a patient with coronavirus disease 2019 (COVID-19) and anosmia [published online ahead of print, 2020 May 29]. JAMA Neurol. 2020. https://doi.org/10.1001/jamaneurol. 2020.2125.

27. Karimi-Galougahi M, Yousefi-Koma A, Bakhshayeshkaram M, Raad N, Haseli S. 18FDG PET/CT scan reveals hypoactive orbitofrontal cortex in anosmia of COVID-19. Acad Radiol. 2020;27:1042-3.

28. Guedj E, Million M, Dudouet P, Tissot-Dupont H, Bregeon F, Cammilleri S, et al. 18F-FDG brain PET hypometabolism in postSARS-CoV-2 infection: substrate for persistent/delayed disorders? Eur J Nucl Med Mol Imaging. (2020 in press).

29. Meles SK, Vadasz D, Renken RJ, et al. FDG PET, dopamine transporter SPECT, and olfaction: combining biomarkers in REM sleep behavior disorder. Mov Disord. 2017;32:1482-6.

30. Jennings D, Siderowf A, Stern M, et al. Conversion to Parkinson disease in the PARS hyposmic and dopamine transporter-deficit prodromal cohort. JAMA Neurol. 2017;74:933-40.

31. Nataf S. An alteration of the dopamine synthetic pathway is possibly involved in the pathophysiology of COVID-19 [published online ahead of print, 2020 Apr 4]. J Med Virol. 2020. https://doi.org/ 10.1002/jmv.25826.

32. Pilotto A, Odolini S, Masciocchi S, et al. Steroid-responsive encephalitis in coronavirus disease 2019 [published online ahead of print, 2020 May 17]. Ann Neurol. 2020. https://doi.org/10.1002/ ana.25783.

33. Morbelli S, Arbizu J, Booij J, et al. The need of standardization and of large clinical studies in an emerging indication of [18F]FDG PET: the autoimmune encephalitis [published correction appears in Eur J Nucl Med Mol Imaging. 2017 Mar;44(3):559-560. Saraf LJ [corrected to Jafari L]]. Eur J Nucl Med Mol Imaging. 2017;44(3):353-7.

34. Grimaldi S, Lagarde S, Harle JR, Boucraut J, Guedj JNM. Autoimmune encephalitis concomitant with SARS-CoV-2 infection: insight from 18F-FDG PET imaging and neuronal autoantibodies. in press

35. Brown EE, Kumar S, Rajji TK, Pollock BG, Mulsant BH. Anticipating and mitigating the impact of the COVID-19 pandemic on Alzheimer's disease and related dementias [published online ahead of print, 2020 Apr 18]. Am J Geriatr Psychiatry. 2020: S1064-748:30294-3.

36. Frank A, Fatke B, Frank W, Förstl H, Hölzle P. Depression, dependence and prices of the COVID-19-crisis. Brain Behav Immun. 2020;87:99. https://doi.org/10.1016/j.bbi.2020.04.068.

37. Li W, Yang Y, Liu ZH, et al. Progression of mental health services during the COVID-19 outbreak in China. Int J Biol Sci. 2020;16: 1732-8.

38. Nobili F, Arbizu J, Bouwman F, et al. European Association of Nuclear Medicine and European Academy of Neurology recommendations for the use of brain $18 \mathrm{~F}$-fluorodeoxyglucose positron emission tomography in neurodegenerative cognitive impairment and dementia: Delphi consensus. Eur J Neurol. 2018;25:1201-17.

39. Moldofsky H, Patcai J. Chronic widespread musculoskeletal pain, fatigue, depression and disordered sleep in chronic post-SARS syndrome; a case-controlled study. BMC Neurol. 2011;11:37.

40. Jo T, Yasunaga H, Michihata N, Sasabuchi Y, Hasegawa W, Takeshima $\mathrm{H}$, et al. Influence of Parkinsonism on outcomes of elderly pneumonia patients. Parkinsonism Relat Disord. 2018;54: 25-9.

41. Papa SM, Brundin P, Fung VSC, et al. Impact of the COVID-19 pandemic on Parkinson's disease and movement disorders. Mov Disord. 2020;35:711-5.

42. Ait Wahmane S, Achbani A, Ouhaz Z, Elatiqi M, Belmouden A, Nejmeddine $M$. The possible protective role of $\alpha$-synuclein against the SARS-CoV-2 infections in patients with Parkinson's disease [published online ahead of print, 2020 Jun 9]. Mov Disord. 2020. https://doi.org/10.1002/mds.28185.

43. Hainque E, Grabli D. Rapid worsening in Parkinson's disease may hide COVID-19 infection. Parkinsonism Relat Disord. 2020: S1353-8020:30117-6.

44. Kuroda N. Epilepsy and COVID-19: associations and important considerations. Epilepsy Behav. 2020;108:107122.

45. Nicol GE, Piccirillo JF, Mulsant BH, Lenze EJ. Action at a distance: geriatric research during a pandemic. J Am Geriatr Soc. 2020;68:922-5.

46. Cummings J, Lee G, Ritter A, et al. Alzheimer's disease drug development pipeline: 2019 Alzheimers. Dement Transl Res Clin Interv. 2019;5:272-93.

47. McDermott MM, Newman AB. Preserving clinical trial integrity during the coronavirus pandemic [published online ahead of print, 2020 Mar 25]. JAMA. 2020. https://doi.org/10.1001/jama.2020. 4689 .

Publisher's note Springer Nature remains neutral with regard to jurisdictional claims in published maps and institutional affiliations. 